FRONTIER ARTICLE

\title{
Orienting polar molecules without hexapoles: Optical state selection with adiabatic orientation
}

\author{
Tim Schäfer ${ }^{\mathrm{a}}$, Nils Bartels ${ }^{\mathrm{a}}$, Nils Hocke ${ }^{\mathrm{a}}$, Xueming Yang ${ }^{\mathrm{b}}$, Alec M. Wodtke ${ }^{\mathrm{a}, \mathrm{c}, \mathrm{d}, *}$ \\ a Institute for Physical Chemistry, Georg-August University of Göttingen, Tammannstr. 6, Göttingen 37077, Germany \\ ${ }^{\mathrm{b}}$ Dalian Institute for Chemical Physics, Dalian, PRC, China \\ ${ }^{\mathrm{c}}$ Department of Dynamics at Surfaces, Max Planck Institute for Biophysical Chemistry, am Faßberg 11, Göttingen, Germany \\ ${ }^{\mathrm{d}}$ Department of Chemistry and Biochemistry, University of California Santa Barbara, Santa Barbara, CA, USA
}

\section{A R T I C L E I N F O}

\section{Article history:}

Available online 22 March 2012

\begin{abstract}
A B S T R A C T
A pedagogic review of technology used to orient polar molecules is presented to place in context the report of a new approach to this problem. Laboratory frame orientation of polar molecules is achieved by state-specific optical pumping in a region free of electric fields followed by adiabatic transport into a static electric field. This approach overcomes some of the limitations of the more common hexapole focusing method. In particular the method is nearly insensitive to the kinetic energy of the sample. We demonstrate production of oriented samples of $\mathrm{NO}\left(\mu_{e l}=0.15 \mathrm{D}\right)$ with translational energies above $1 \mathrm{eV}$ in both high- and low-field seeking states. The method can be extended to many other classes of molecules, including near symmetric tops and ions.
\end{abstract}

(c) 2012 Elsevier B.V. All rights reserved.

\section{Introduction}

The spatial configuration of atoms serves as well as any as a definition for molecular identity, structure and, to a large extent, function. In direct analogy, how a molecule's atoms are spatially configured with respect to its surroundings helps to define its functional interactions with its environment (see Figure 1). This statement helps explain the long standing motivation to develop means of controlling molecular orientation; that is to say: controlling how a molecule points in real space.

Nowhere has the desire to understand orientational impacts on function driven scientific investigation more than in the field of surface chemical dynamics [1-6]. In addition to the following reviews, to which the reader is directed $[7,8]$, specific examples of studies include orientational influences on rotational inelastic energy transfer in molecule-surface collisions $[1,2]$. In addition, orientation influences sticking at surfaces. The sticking probability of $\mathrm{CH}_{3} \mathrm{Cl}$ on $\mathrm{Si}(100)$ was observed to be higher for a ' $\mathrm{Cl}$-end collision' in comparison to a ' $\mathrm{CH}_{3}$-end collision' [3]. In other work, it was seen that for an 'N-end collision' of NO on $\mathrm{Si}(111)(7 \times 7)$, dissociation is enhanced [4]. NO sticking on $\mathrm{Ni}(100)$ is similarly enhanced for 'N-end collisions' [5]. Electronically nonadiabatic molecule-surface interactions [9-12] have also been reported to exhibit orientational influences. Enhanced electron exoemission

\footnotetext{
* Corresponding author at: Institute for Physical Chemistry, Georg-August University of Göttingen, Tammannstr. 6, Göttingen 37077, Germany.

E-mail address: alec.wodtke@mpibpc.mpg.de (A.M. Wodtke).
}

was observed for 'O-end collisions' of vibrationally excited $\mathrm{N}_{2} \mathrm{O}$ on Cs adlayers on $\mathrm{Pt}(111)$ [6].

Orientational effects are also thought to be important in electron transfer chemistry. Indeed, the authors of Ref. [6] invoked an orientational influence on harpooning [13-15] to explain their observations. Recently, a new ab initio theory of electronically nonadiabatic molecule-surface interactions [16,17] has predicted a strong steric effect for the vibrational relaxation of highly vibrationally excited NO. Specifically, experiments from our laboratory employing un-oriented $\mathrm{NO}(v \gg 0$ molecules in collisions with $\mathrm{Au}(111)$ reported multiquantum vibrational relaxation mediated by electron transfer [18]. The $a b$ initio theory predicted energy transfer (and hence the electron transfer) is completely suppressed for O-end collisions [19]. In order to test the assumptions underlying this theory, we were led to consider means for producing oriented samples of NO in high vibrational states using stimulated emission pumping $[20,21]$ with variable kinetic energies between about 30 and $1000 \mathrm{meV}$. The reason we targeted this range of translational energies of incidence - steering effects - is itself informative to the consideration of orientation experiments. Steering effects describe a class of phenomena where oriented molecules become reoriented on their approach to their collision partner [3,22-25]. If one wishes to see the effect of orientation on a collision, it is necessary that the molecules initial orientation 'be remembered' during the collision. Steering effects can erase this memory. One way to overcome steering effects is to reduce the time the molecules spend in the entrance channel to the collision partner, i.e. increase the translational energy of incidence. 


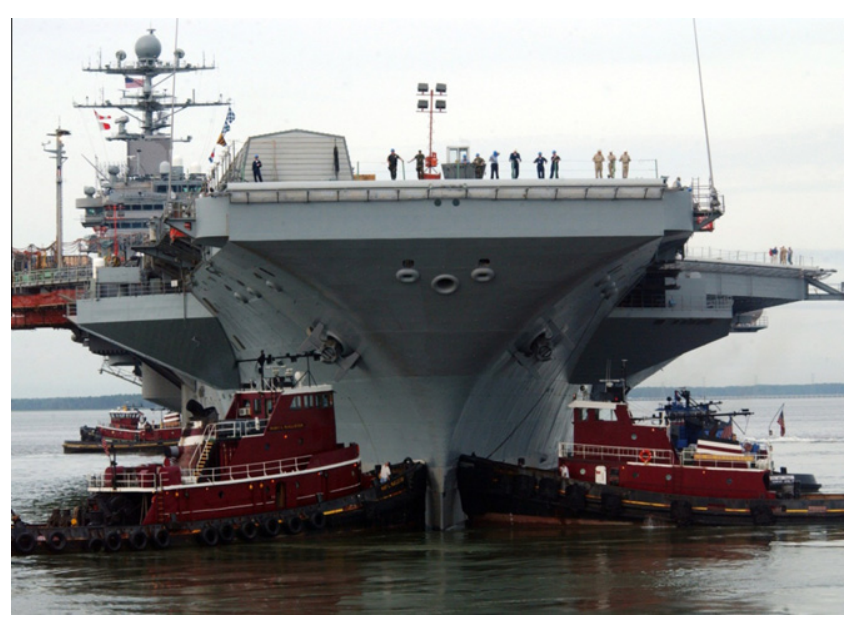

Figure 1. Influence over orientation is necessary to proper function, in this case docking.

In the course of designing an orientation experiment to study steric effects in molecule-surface collisions, we were confronted with some of the limitations of established methods for orientation, especially as they pertain to samples with high translational energies of incidence. We now explain some of the theory behind conventional orientation methods to make these limitations clearer.

\section{The basics of traditional orientation methods}

For polar molecules, applying an external electric field exerts a force that is capable of orienting the molecule with respect to the direction of the field. Furthermore, by varying the direction of the electric field with respect to a fixed direction in the laboratory frame of reference, one may alter the direction the molecule points in space. One of the most successful approaches to orienting polar molecules has been to selectively focus specific rotational states with quadrupole [26] and hexapole [27] lenses. These devices not only select individual quantum states of molecules, but in addition, those states are oriented (or aligned) and introduced to a subsequent orienting electric field. The orientation (or alignment produced by the state selecting lens) is preserved or redirected by the orientation field for use in the experiment (see Figure 2).

\subsection{Quadrupole lenses}

Quadrupole lenses (see Figure 3a) are subject to the properties of the (2nd order and higher) Stark effect for linear molecules in $\Sigma$ states.
$\Delta W_{\text {Stark }, \Sigma}=\frac{\mu_{e l}^{2} E^{2}}{2} \frac{2 I}{h^{2}} \frac{J(J+1)-3 M^{2}}{J(J+1)(2 J-1)(2 J+3)}+\mu_{e l}^{4} E^{4}$

Here, $\mu_{e l}$ is the dipole moment of the molecule, $E$ is the strength of the electric field, $I$ is the molecule's moment of inertia, $\hbar$ is the reduced Planck's constant, and $J$ and $M$ are rigid rotor quantum numbers. For effective focusing, we next discard terms higher than 2nd order and consider only molecules with specific quantum numbers. Namely, for $J>0, M=0$, the equation simplifies to the following

$\Delta W_{\text {Stark }, \Sigma}=\frac{\mu_{e l}^{2} E^{2}}{2} \frac{2 I}{h^{2}} \frac{1}{4 J(J+1)-3}$

Electric quadrupole fields have the property that $E \propto r$ where $r$ is the distance from the symmetry axis of the quadrupole (see Figure 3a). Hence, if one passes a divergent molecular beam along the symmetry axis of a quadrupole electric field, the molecules in the states we are considering experience a parabolic transverse potential. This induces a sinusoidal - and naturally refocusing - trajectory and the effect is that of a lens with a focal length, $\ell$.

$\ell_{q}=\sqrt{\frac{1 / 2 m v^{2}}{4^{V_{q}^{2}} / r_{0}^{4} \mu_{e l}^{2}}[4 J(J+1)-3]}$

Here, $V_{q}$ is the potential applied to the quadrupole rods, $r_{0}$ is the closest distance of the rod to the symmetry axis, $m$ is the mass of the molecule, and $v$ is its velocity. By placing an aperture in the focal plane of the lens, individual $J$ states - all with $M=0$, may be selectively transmitted. At a fixed value of $V_{q}$ the focal length, $\ell$, scales approximately linearly with $J$ a fact that when combined with the low rotational temperatures typical of molecular beams, often imposes a practical constraint in many experiments that only the $J=1, M=0$ state can be usefully refocused.

It is possible to refocus states with $|M|>0$ - See for example Ref. [28] - and a generalization to the focal length formula can be derived (within second order in $E$ ).

$\ell_{q, g e n}=\sqrt{\frac{1 / 2 m v^{2}}{4^{V_{q}^{2}} / r_{0}^{4} \mu_{e l}^{2}} \frac{h^{2}}{2 I}\left[\frac{J(J+1)(2 J-1)(2 J+3)}{J(J+1)-3 M^{2}}\right]}$

It is important, however, to realize that most states with $|M|>0$, will be defocussed, i.e. $\ell_{q, g e n}$ is imaginary. Additionally, orientation cannot occur for the $M=0$ states that are easily refocused by the quadrupole and even for the $|M|>0$ states that can be refocused, the quadrupole lens does not distinguish the sign of $M$. Thus in practice the quadrupole is only useful for preparing aligned samples of molecules.

Despite some of these limitations, the quadrupole has been used extensively in molecular physics experiments. For example,

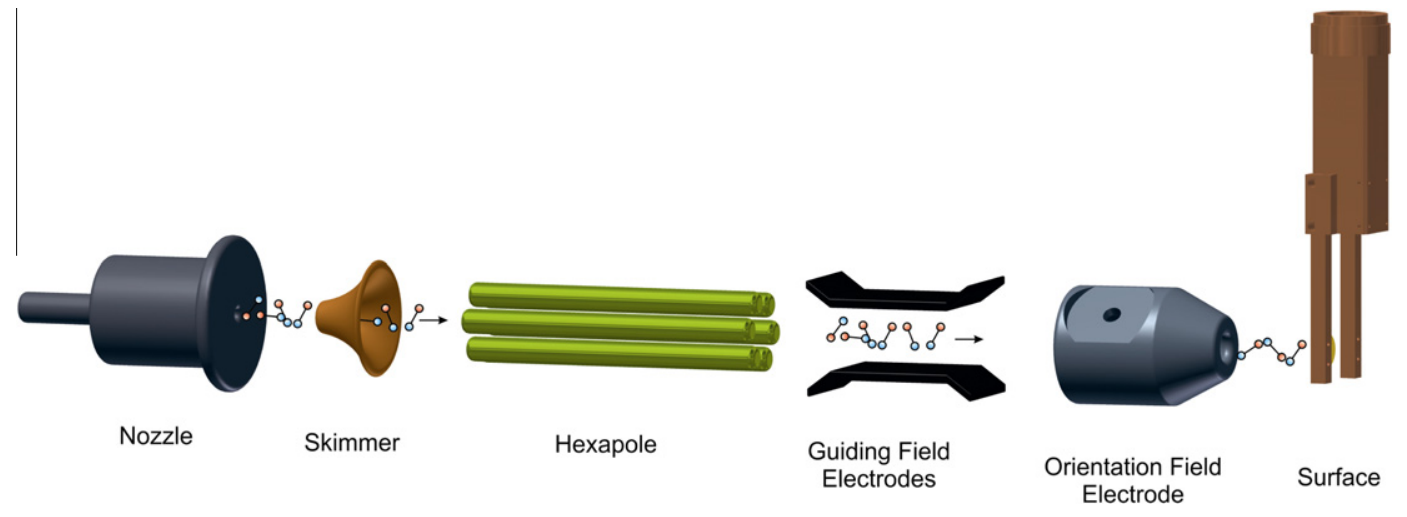

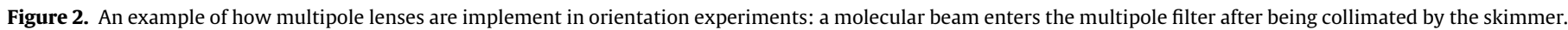

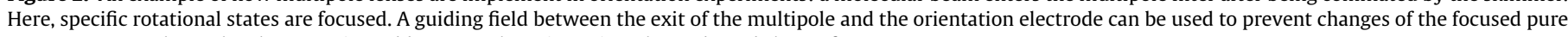
quantum state. The molecules are oriented between the orientation electrode and the surface. 

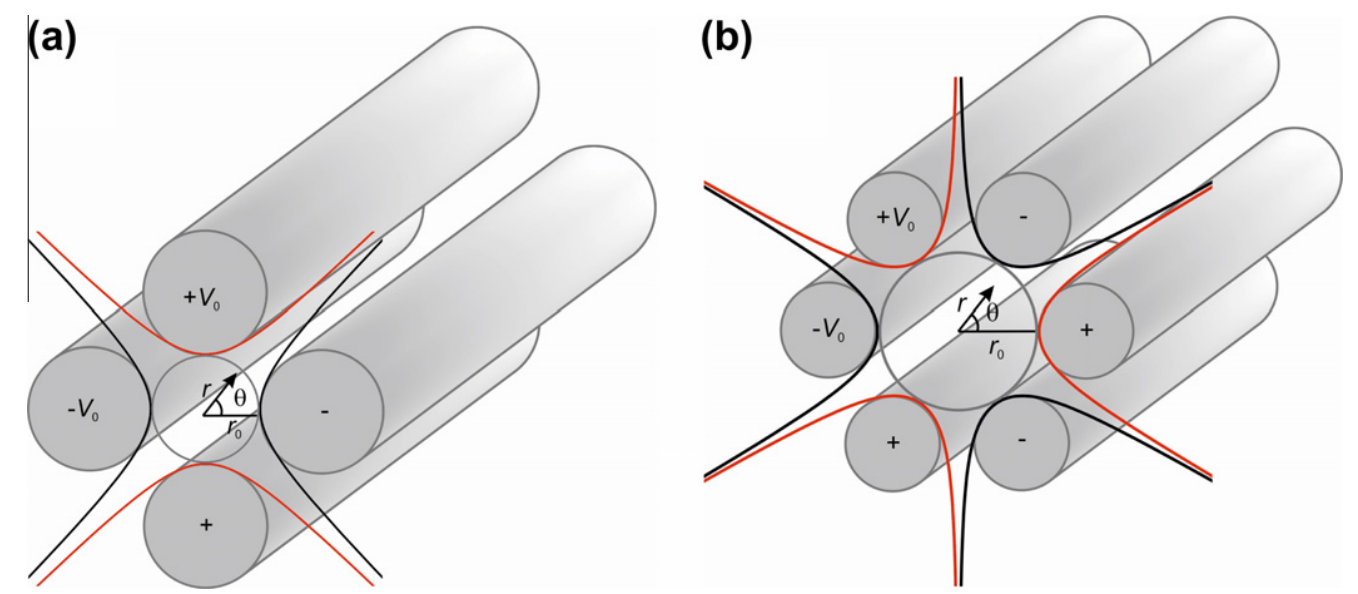

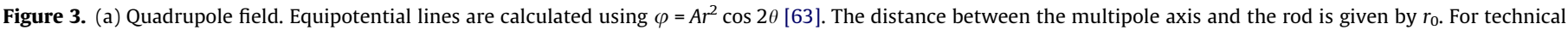

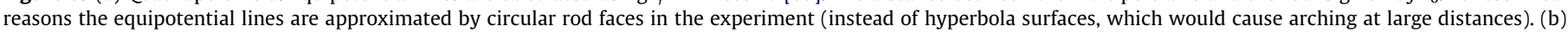
Hexapole field. Equipotential lines are calculated using $\varphi=A r^{3} \cos 3 \theta$ [63]. Again, for technical reasons circular rods are commonly used.

it has been used to investigate the angular dependence of dipoleinduced dipole interactions between TIF and rare gases [29].

\subsection{Hexapole lenses}

The situation becomes more interesting for the case of hexapole fields (see Figure 3b). Here the 1st order Stark effect for symmetric top molecules with rotational quantum numbers $J, K$ and $M$ can be exploited [27].

$\Delta W_{\text {Stark }}^{(1)}=-\mu_{e l} E\langle\cos \vartheta\rangle_{\max }$

where, $\langle\cos \vartheta\rangle_{\max }=\frac{K M}{J(J+1)}$

A hexapole field produces an electric field $E \propto r^{2}$. In an analogous fashion to the above discussion sinusoidal refocusing trajectories can arise for specific states of symmetric top molecules passing along the symmetry axis of the hexapole. The hexapole then acts as a lens, with focal length, $\ell_{\text {hex }}$.

$\ell_{\text {hex }}=\sqrt{\frac{\pi^{2} r_{0}^{3}}{3} \frac{J(J+1)}{M K} \frac{\frac{1}{2} m v^{2}}{\mu_{e l} V_{\text {hex }}}}$

\subsection{Symmetric top states within and without electric fields}

In contrast to quadrupoles, hexapoles easily produce oriented states of molecules. The rotational states of symmetric tops can be written as follows [30].

$|J, K, M\rangle=\sqrt{\frac{(2 J+1)}{8 \pi^{2}} \mathcal{D}_{K, M}^{J}(\chi, \theta, \phi)}$

Where $\mathcal{D}_{K, M}^{I}(\chi, \theta, \phi)$ are the Wigner rotation matrices, $\theta$ is the polar angle with respect to the electric field, $\varphi$ is the azimuthal angle and $\chi$ is the internal azimuthal angle describing the rotation about the symmetric top axis. Eigenstates of parity relevant to experiments in field free conditions are constructed thus.

$|J| K,|, M, \pm\rangle=\frac{1}{\sqrt{2}}(|J, K, M\rangle+\varepsilon|J,-K, M\rangle)$

here, $\varepsilon= \pm 1$ defines the parity of the state and is labeled with $e(\varepsilon=+1))$ and $f(\varepsilon=-1))$. In a strong electric field of a hexapole, the parity states mix producing orientation states. $\left|\varphi_{ \pm}\right\rangle_{E}=\frac{1}{\sqrt{\alpha(E)^{2}+\beta(E)^{2}}}(\alpha(E)|J| K,|, M,+\rangle \pm \beta(E)|J| K,|, M,-\rangle)$

Here, the mixing coefficients, $\alpha(E), \beta(E)$ depend on the strength of the electric field, $E$. In the limit of strong mixing, $\alpha(E)=\beta(E)=\frac{1}{\sqrt{2}}$ the orientation states reduce to the following form.

$\left|\varphi_{ \pm}\right\rangle_{E \rightarrow \infty}=\frac{1}{\sqrt{2}}(|J| K,|, M,+\rangle \pm|J| K,|, M,-\rangle)$

Such states are strongly oriented in the electric field created by the hexapole; the degree of orientation being limited only by the Heisenberg uncertainty principle. The orientation angle can be accurately estimated from Eq. (6), $\langle\cos \varphi\rangle_{\max }=\frac{K M}{J(I+1)}$. Alternatively, the wave functions can be integrated to find expectation values. For example, strong field limited orientation states of the NO molecule with $J=\frac{1}{2}, K=\frac{1}{2}, M=\frac{1}{2}$ have an average angle with respect to $E$ of $67.5^{\circ}$ and $112.5^{\circ}$, respectively [31]. One of these states is oriented with $\mu_{e l}$ antiparallel to $E$; that is, energetically unfavorably and feels a restoring force driving it back to the symmetry axis of the hexapole, where $E$ is minimum (low field seeking state). The other state is oriented with $\mu_{e l} \| E$; that is, energetically favorably and is defocussed to regions of strong electric field (high field seeking states) and is not delivered to the output of the hexapole.

\subsection{Limitations of the multipole lens approach}

Despite the undisputed success of the multipole lens approach, a conscientious student will already begin to see serious limitations upon reflecting on some of the equations presented above. To make this clearer, consider the case of hexapole focusing of a polar symmetric top molecule. As shown above, the focal length of hexapoles scales with the kinetic energy of the beam. This can be rewritten as follows:

$l_{\text {hex }}=\sqrt{\frac{\pi^{2} r_{0}^{3}}{3\langle\cos \vartheta\rangle_{\max }} \frac{1 / 2 m v^{2}}{\mu_{e l} V_{\text {hex }}}} \propto \sqrt{\frac{\text { kinetic energy }}{\text { Stark energy }}}$

This has two major consequences. First, it means that perfect focusing is only possible for molecules of a single kinetic energy. Hence, the hexapole state resolving power is rapidly reduced by any velocity spread in the sample and may even require use of a velocity selector [26,27]. Second, the Stark Energy is typically as small as $10^{4} \mathrm{eV}$. Kinetic energies of molecular beams can easily be as large as $1 \mathrm{eV}$. Consequently, especially for molecules with small dipole moments, only modest kinetic energies can be refocused [32-34]. 
For example, the NO molecule has been commonly used for hexapole-based orientation experiments, where a hexapole of $1 \mathrm{~m}$ length is used to refocus beams with $0.2 \mathrm{eV}$ kinetic energy. Refocusing beams with only $1 \mathrm{eV}$ kinetic energy would require hexapoles of more than twice this length. Yet, chemical studies of oriented molecules at high kinetic energy are especially interesting, since reorienting forces in chemical reactions, steering effects [3,22-25] can be overcome.

The kinetic energy spread degraded rotational resolving power of hexapoles also makes it nearly impossible to orient more complex polyatomic molecules, especially if they are asymmetric tops. See for example work on formaldehyde $[35,36]$. In this case the molecular beam typically produces a sample with several populated rotational states that cannot be resolved by the hexapole [37].

The fact that only certain choices of rotational quantum numbers can be refocused represents another limitation of this approach. First of all, states that are defocused by the hexapole - termed high-field seekers - may be of interest to the experimentalist. Secondly, since only low field seekers can be refocused by the hexapole, switching large electric orientation fields located after the hexapole is needed to change the orientation of the low-field seeker in the experiment.

\section{Field-free optical pumping followed by adiabatic orientation: a new approach}

As described in the last section, traditional orientation experiments consist of a state selector (quadrupole or hexapole) followed by an orientation field that might also be used to re-orient the molecule before the experiment is carried out. Reorientation is possible if the electric field is large enough and varies slowly enough that transitions between the Stark split levels are not induced. This requirement is rather easy to meet under most realistic experimental conditions.

It is fundamentally important to understanding this Letter that one realizes that in many molecules, defined parity states are energetically split in zero-field due to internal dynamical effects (see Figure 4). An example is $\Lambda$-doubling in NO. This begs the question: 'Can one use laser optical excitation to prepare single parity levels in a field free region and let them fly adiabatically into an orienting electric field?' If so, these parity states will be mixed as they enter the orientation field adiabatically correlating to orientation states. Following a non-crossing rule, the low energy member of the field free doublet will correlate to the high field seeking state and the high energy member will transform to the low field seeking state. This concept is similar to previous work where lasers were used to optically excite individual M-states, whose degeneracy was broken by an external electric field [38]. We take the idea of Ref. [38] one step further, effectively lifting the M-state degeneracy via the internal dynamics of the molecule itself.

In this Letter, we show that optical pumping is indeed an attractive alternative to hexapole state selection, offering solutions to many of the problems of multipole lenses. Here, specific rotational states prepared by laser excitation are directly injected into an orientation field. Under the adiabatic condition, the laser prepared states are transformed into quantum mechanically optimal orientation states for use by the experimentalist. In contrast to the multipole lens approach, the methods demonstrated here are essentially insensitive to the kinetic energy of the molecular sample. Not only may one work with samples with broad kinetic energy distributions, high kinetic energies are also easily oriented even for molecules with small dipole moments. Another advantage stems from the fact that the electrode needed for orientation is small compared to a multipole lens. Thus, the distance from the source to the experiment can be reduced, vastly simplifying the apparatus. This method is also capable of orienting both low field and high field seeking states. This allows rapid optical switching between orientation states using a static orientation field. Finally, theoretical analysis shows that this method is applicable to asymmetric top molecules with complex rotational structure.

The remainder of the Letter is organized as follows. First, we describe our experimental apparatus and the results obtained for the orientation of NO. Based on our theoretical understanding of the NO case, we then elaborate upon how this technique can be applied more generally, including applications to linear molecules, symmetric tops, asymmetric tops and even ions.

\subsection{Experimental details}

Specifically in this Letter, we demonstrate optical state selection with adiabatic orientation. Using stimulated emission pumping (SEP) of NO, we prepare single parity states ( $e$ or $f$ ) in the rotational level of the $X^{2} \Pi_{1 / 2}\left(v^{\prime \prime}=16, J=1 / 2\right)$ state in a field free region. The molecules then fly into an electric field and we follow the adiabatic re-coupling producing oriented NO by laser induced fluorescence spectroscopy as a function of the strength of the external electric field.

The experiments are carried out in a molecular beam apparatus similar to that described in previous papers $[39,40]$. A pulsed supersonic molecular beam of rotationally cold NO molecules $\left(T_{R O T} \sim 6 \mathrm{~K}\right)$ is produced by expanding either a $60 \% \mathrm{NO} / \mathrm{Kr}(\mathrm{KE}=0.035 \mathrm{eV})$ or a $1 \%$ $\mathrm{NO}$ in $\mathrm{H}_{2}(\mathrm{KE}=1.0 \mathrm{eV})$ mixture into the vacuum through a piezoelectric valve ( $1 \mathrm{~mm} \varphi$ nozzle, $10 \mathrm{~Hz}, 3 \mathrm{Atm}$. stagnation pressure). After passing a $2 \mathrm{~mm}$ electro-formed skimmer (Ni Model 2, Beam

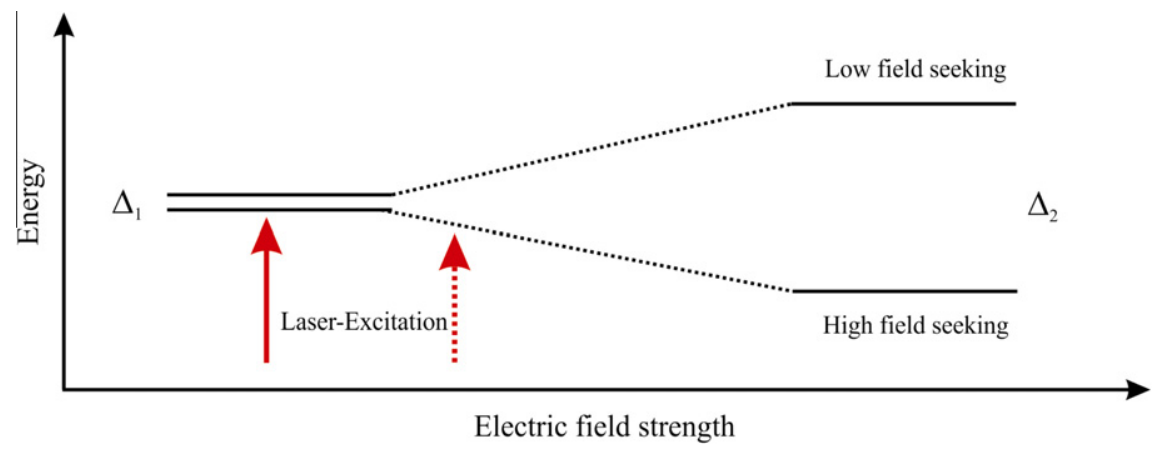

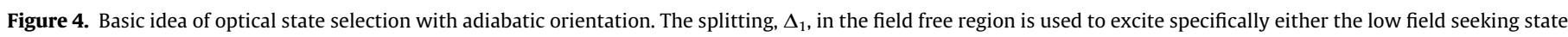

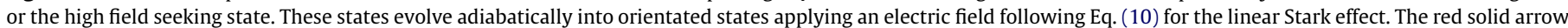
denotes the excitation in the field free region. If more splitting or a quantization axis is needed excitation can take place in the electric field (dotted arrow). 
dynamics, Inc.) $3 \mathrm{~cm}$ downstream, the beam enters a differentially pumped region, where stimulated emission pumping $[20,21]$ (SEP) is carried out. Populations of $\mathrm{NO} X^{2} \Pi_{1 / 2}\left(v^{\prime \prime}=16\right)$ in single parity levels of the $J=1 / 2$ state are produced by exciting the $B^{2} \Pi_{1 / 2}\left(v^{\prime}=3\right) \leftarrow X^{2} \Pi_{1 / 2}\left(v^{\prime \prime}=0\right) R_{11}\left(\frac{1}{2}\right)$ (PUMP) transition followed by de-exciting the $B^{2} \Pi_{1 / 2}\left(v^{\prime}=3\right) \rightarrow X^{2} \Pi_{1 / 2}\left(v^{\prime \prime}=16\right) R_{11}\left(\frac{1}{2}\right)$ (DUMP) transition. The PUMP step requires light at $206.15 \mathrm{~nm}$, produced by a narrow bandwidth homebuilt OPO-SFG laser system $(250 \mathrm{MHz}$ resolution, $1 \mathrm{~mJ} /$ pulse) [41]. The PUMP step is carried out in a field free region where parity selection rules are strongly obeyed. The high resolution OPO-SFG light source is capable of resolving the $R_{11}\left(\frac{1}{2}\right) \Lambda$-doublet transitions (see Figure 5 ). This produces states of defined parity ( $e$ or $f$ ). Stimulated emission transfers population to the NO $X^{2} \Pi_{1 / 2}\left(v^{\prime \prime}=16, J=\frac{1}{2}\right)$ state, preserving the parity selected in the PUMP step. This is carried out using the $458.1 \mathrm{~nm}$ output of a Nd:YAG pumped (PRO-270, Spectra Physics) dye laser (PRSCDA-24, Sirah) with a pulse energy of $8 \mathrm{~mJ}$ and a bandwidth of $3000 \mathrm{MHz}$. The SEP preparation is monitored by laser induced fluorescence (LIF) and fluorescence depletion spectroscopy using a quartz lens and a photomultiplier tube (PMT, Hamamatsu, R7154) [42].

The state-specifically prepared NO molecules are in single parity levels of $X^{2} \Pi_{1 / 2}\left(v^{\prime \prime}=16, J=\frac{1}{2}\right)$ and fly downstream in the molecular beam into another differentially pumped vacuum chamber and pass along the symmetry axis of a cylindrical $(7 \mathrm{~cm}$ long) stainless steel electrode positioned $1 \mathrm{~cm}$ in front of a flat grounded $\mathrm{Au}(111)$ sample, which is used to apply electric fields up to $18 \mathrm{kV} /$ $\mathrm{cm}$ parallel to the propagation axis of the molecular beam (see Figure 6). The $\mathrm{Au}(111)$ surface is located $17 \mathrm{~cm}$ downstream from the SEP state preparation zone. The $450 \mathrm{~nm}$ output of a Nd:YAG pumped (Powerlite 7010, Continuum) dye laser (CSTR-DA-24, Sirah) is used to detect the $\mathrm{NO}(v=16)$ molecules in the orientation field by resonantly exciting the molecules to the $A^{2} \Sigma(v=2)$ state and monitoring the LIF on a PMT (Hamamatsu, R7154). For detection of the PMT signals we utilize a digital oscilloscope (LT344, LeCroy) interfaced to a computer.

The strength of the orientation field averaged over the $\left(\sim 1 \mathrm{~mm}^{3}\right)$ probe volume located between the cylindrical electrode and the gold surface is determined by measuring the Stark splitting of the $B^{2} \Pi_{1 / 2}\left(v^{\prime}=3\right) \leftarrow X^{2} \Pi_{1 / 2}\left(v^{\prime \prime}=0\right) R_{11}\left(\frac{1}{3}\right)$ PUMP transition as a function of the electric field. Since all molecular constants needed

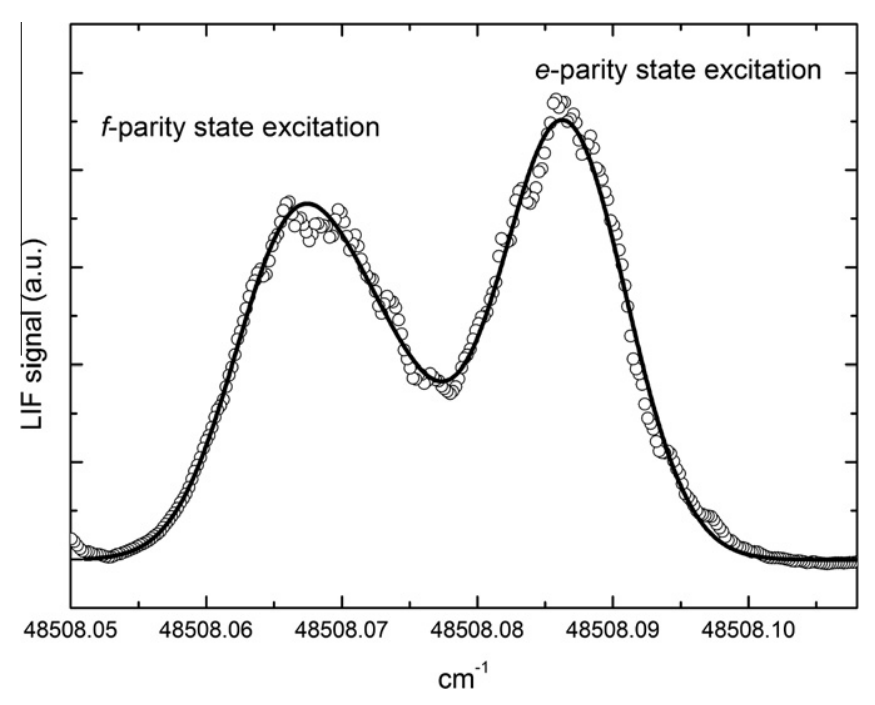

Figure 5. LIF spectrum of the $B^{2} \Pi_{1 / 2}(v=3) \leftarrow X^{2} \Pi_{1 / 2}(v=0) R_{11}(1 / 2)$ line. The spectrum displays the $\Lambda$-doubling, which is used to excite different $e$ or $f$-parity states.

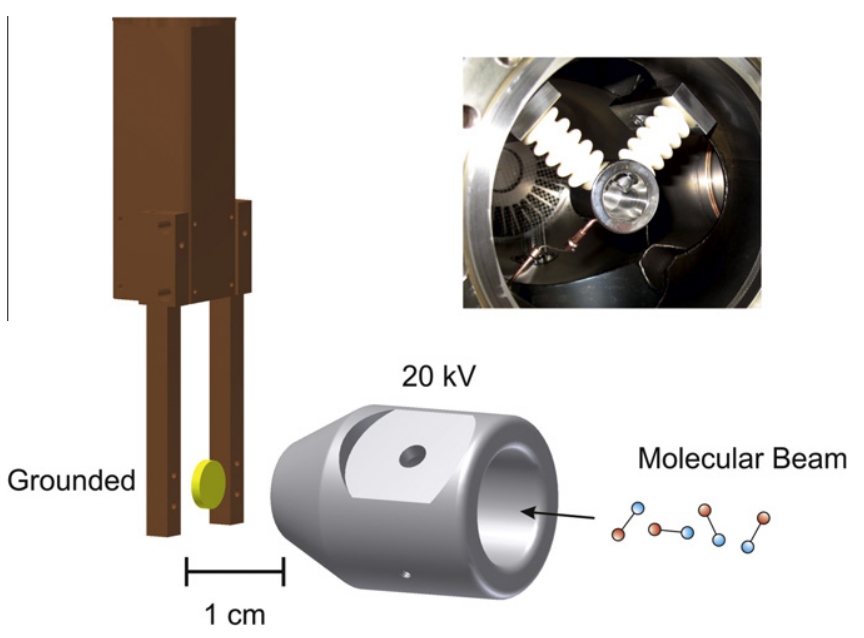

Figure 6. Simple experiment: the orientation electrode is mounted $1 \mathrm{~cm}$ in front of the surface, capable to produce fields up to $20 \mathrm{kV} / \mathrm{cm}$. The probe laser excites the NO molecules between electrode and surface.

to calculate these splitting are known [43], the observed splitting can be used to derive the strength of the electric field.

\subsection{Results}

As explained above, we use SEP with a narrow bandwidth PUMP laser to prepare a population of $\mathrm{NO}^{2} \Pi_{1 / 2}\left(v=16, J=\frac{1}{2}\right)$ in a pure parity state described by Eq. (9). This population adiabatically enters a region with a large electric field, mixing the parity levels and transforming the zero field state into one of the orientation states described by Eq. (10). The degree of orientation in the electric field can be deduced by spectroscopic methods following the work of de Lange et al. [44]. This method relies on the parity selection rules

$$
\begin{aligned}
& e \leftrightarrow e \\
& f \leftrightarrow f \\
& +\leftrightarrow- \\
& e \leftrightarrow f \\
& +\leftrightarrow+ \\
& -\leftrightarrow-
\end{aligned}
$$

These selection rules give rise to strong and weak lines in the PROBE spectrum under field free conditions. If the parity state purity is perfect, weak lines are completely absent. As an electric field is applied, the parity states mix and the intensities of strong and weak lines changes, where strong lines become weaker and weak lines become stronger. By measuring intensity ratio's, one may directly extract the mixing coefficients: $\alpha(E)$ and $\beta(E)$.

Figure 5 shows the LIF spectrum of the PUMP transition used in this Letter: $B^{2} \Pi_{1 / 2}\left(v^{\prime}=3\right) \leftarrow X^{2} \Pi_{1 / 2}\left(v^{\prime \prime}=0\right) R_{11}\left(\frac{1}{2}\right)$. The home-made laser system is able to clearly resolve the $\Lambda$-doublets, which are separated by about $600 \mathrm{MHz}$. However, due to the underlying hyperfine structure and residual Doppler width, the parity state selection is not perfect. We will presently show that there is a $15 \%$ contamination from the 'wrong parity state', which can easily be accounted for in the analysis and has no substantive impact on the meaning or interpretation of the experimental results. Furthermore, by tuning the PUMP laser one can easily switch between states of opposite parity. This is made simpler by the fact that the wavelength of our laser system is defined by a diode laser that can be rapidly scanned with external electronic control.

The DUMP laser transfers population to the $X^{2} \Pi_{1 / 2}\left(v^{\prime \prime}=16, J=\right.$ $\left.\frac{1}{2}\right)$ state. Despite its broad bandwidth, the parity selection 


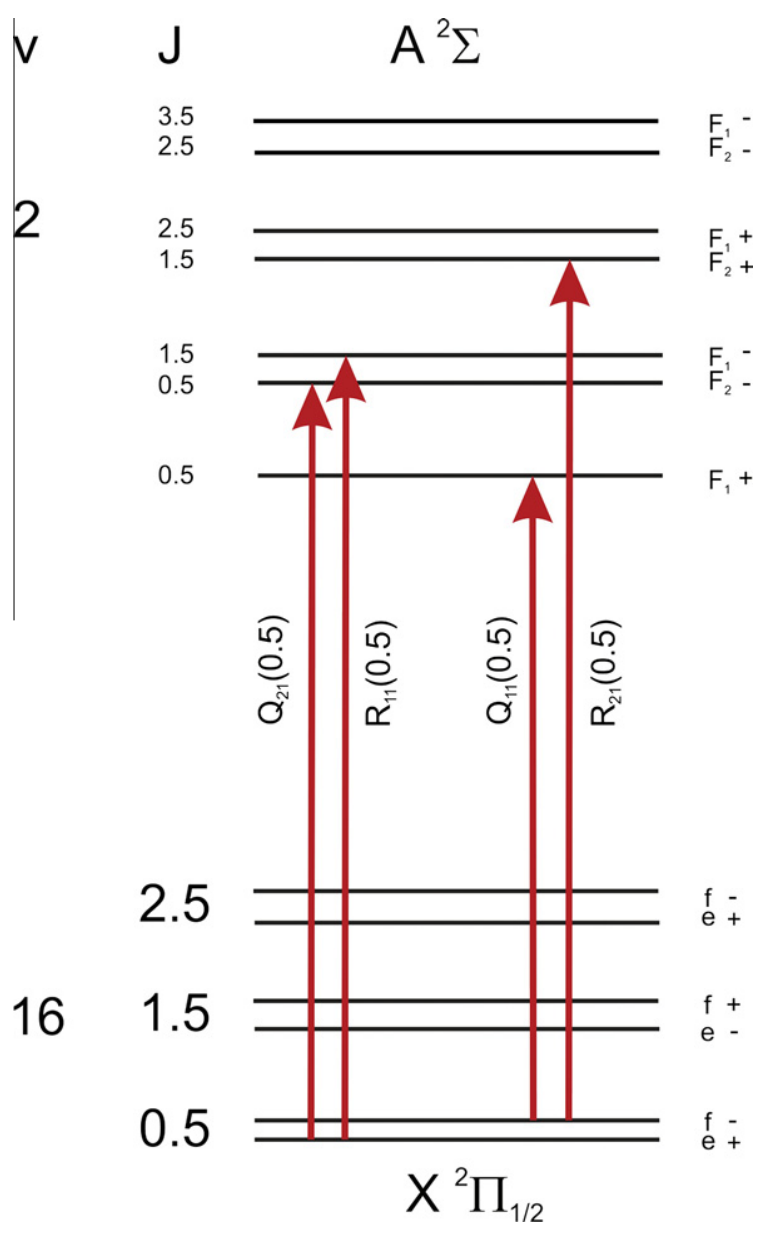

Figure 7. Energy diagram and symmetry labels of the transition $A^{2} \Sigma(v=2) \leftarrow X^{2} \Pi_{1}$ ${ }_{2}(v=16)$ relevant to probe the degree of orientation in the electric field. When preparing NO molecules in an e-parity state, the left arrows display the observed transitions. When preparing NO molecules in a $f$-parity state the transitions on the right side are observed.

rules - Eq. (13) - are obeyed, which means the DUMP step switches the parity selected by the PUMP laser.

Figure 7 shows the energy diagram and symmetry labels of the lowest rotational states of the $X^{2} \Pi_{1 / 2}\left(v^{\prime \prime}=16\right)$ level and all possible transitions that can be seen via the PROBE band: $A^{2} \Sigma_{1 / 2}\left(v^{\prime}=2\right) \leftarrow X^{2} \Pi_{1 / 2}\left(v^{\prime \prime}=16\right)$. The parity of each level is also shown in Figure 7. The doubling in the $A^{2} \Sigma_{1 / 2}$ state - labeled $\mathrm{F}_{1} / \mathrm{F}_{2}$ - is extremely small due to the spin-rotation interaction $\left(\gamma=2.71810^{-3} \mathrm{~cm}^{-1}\right)$ [45]. Here, the parity changes only with the $\mathrm{N}$ quantum number, meaning there are large (several $\mathrm{cm}^{-1}$ ) energy differences between nearest states of opposite parity. In the absence of an electric field, the $A^{2} \Sigma_{1 / 2}\left(v^{\prime}=2\right) \leftarrow X^{2} \Pi_{1 / 2}\left(v^{\prime \prime}=16\right)$ LIF PROBE spectrum depends sensitively on the parity of the state prepared by SEP. Specifically in the case of a pure parity state, one observes either a single overlapped $R_{11}(0.5) / Q_{21}(0.5)$ line (in the case of $e$ parity state preparation) or two well-resolved lines separated by nearly $10 \mathrm{~cm}^{-1}: R_{21}(0.5)$ and $Q_{11}(0.5)$ (in the case of $f$ parity state preparation) (see Figure 7 ). Figure $8 \mathrm{a}$ and $\mathrm{c}$ show the corresponding PROBE spectra in the absence of an external electric field. Due to imperfections in the preparation method, one observes a small contribution from the unwanted parity state.

When turning on the electric field, zero field forbidden transitions become allowed due to the mixing of the zero field wave functions. These transitions increase in intensity with increasing E. Similarly, the allowed transitions loose intensity. This is shown in Figure $8 \mathrm{~b}$ and $\mathrm{d}$. Here, an electric field of $18 \mathrm{kV} / \mathrm{cm}$ is present at the position where the PROBE laser crosses the molecular sample. The $2: 1$ intensity ratio of the $Q_{11}(0.5)$ to the $R_{21}(0.5)$ peak reflects the Hönl-London factors of these transitions. It clearly indicates, that the transitions are not saturated, a fundamental condition necessary to deduce the degree of orientation from the line intensities in the LIF spectrum [44].

The mixing coefficients, $\alpha(E)$ and $\beta(E)$, are derived from the experimental data by simulating the LIF-spectra with appropriate Voigt profile functions. The spectral contributions of both parity states are weighted according to the line intensities in the zerofield spectra.

$\sigma(E)=(1=\tau) \sigma_{1}(E)+\tau \sigma_{2}(E)$

Here, $\sigma(E)$ describes the overall LIF-spectrum, $\sigma_{1}(E)$ is the contribution to the spectrum from the optically prepared parity states, $\sigma_{2}(E)$ is the contribution from the other (impurity) parity state. A best fit to the zero field spectrum is obtained for a value of $\tau=0.15$, which describes the magnitude of the impurity.

$\sigma_{1}(E)$ and $\sigma_{2}(E)$ are modeled using fixed line positions with the ratio of the intensities given by the known Hönl-London factors, so that the mixing coefficient $\beta(E)^{2}$ remains the only fitting parameter.

$$
\begin{aligned}
& \sigma_{1}(E)=\left(1=\beta(E)^{2}\right) \sigma_{f}+\beta(E)^{2} \sigma_{e} \\
& \sigma_{2}(E)=\beta(E)^{2} \sigma_{f}+\left(1-\beta(E)^{2}\right) \sigma_{e},
\end{aligned}
$$

Here, $\sigma_{f}$ and $\sigma_{e}$ are the spectra originating from the $e$ and $f$ parity state, respectively. Of course, $\alpha(E)^{2}$ is obtained easily, since the mixing coefficients are normalized.

Figure 9 shows the corresponding simulations for electric fields of 0 and $18 \mathrm{kV} / \mathrm{cm}$. In Figure 9 the data are given as solid points. The fit to the data using an optimized value of $\beta(E)^{2}$ is shown as a black solid line. The red line shows the contribution from the targeted parity state and the blue line shows the contribution from the impurity parity state.

Knowledge of the mixing coefficients allows us to calculate the degree of orientation of the NO molecule achieved in this experiment, since the mixing coefficients, $\alpha(E)$ and $\beta(E)$, fully describe the degree of orientation [44]. Specifically one may employ the following formula.

$\langle\cos \vartheta\rangle_{E}=2 \alpha(E) \beta(E)\langle\cos \vartheta\rangle_{\max }$

Here, $\vartheta$ is the angle between the electric field and the molecular axis of the NO molecule and $\langle\cos \vartheta\rangle_{\max }$ is defined by Eq. (6).

Systematically analyzing a series of spectra like this at many different values of $E$ provides the mixing as a function of applied electric field. Figure 10 shows these results presented in two ways, $\beta(E)^{2}$ and $2|\alpha(E) \beta(E)|$, as a function of field strength. The degree of mixing at the maximum electric field $(18 \mathrm{kV} / \mathrm{cm})$ is nearly complete. Furthermore the results are independent of the kinetic energy of the sample as can be seen in Figure 10, where data at 45 (black circles) and $1000 \mathrm{meV}$ (red stars) is shown.

As further evidence of the validity of this analysis, one may calculate $\beta(E)^{2}$ and $2|\alpha(E) \beta(E)|$, using the molecular constants of NO for the vibrational state under consideration using the formulas presented in Ref. [44].

$$
\begin{aligned}
& \alpha(E)=\sqrt{\frac{1}{2}+\frac{1}{2 \sqrt{1+E_{\text {Red }}^{2}}}} \\
& \beta(E)=\sqrt{\frac{1}{2}-\frac{1}{2 \sqrt{1+E_{\text {Red }}^{2}}}}
\end{aligned}
$$

Here, $E_{\text {Red }}$ is the ratio of twice the Stark energy to the zero field $\Lambda$ splitting: $\frac{2 \Delta W_{\text {Stark }}^{(1)}}{W_{\Lambda}}$. 
e-parity selected

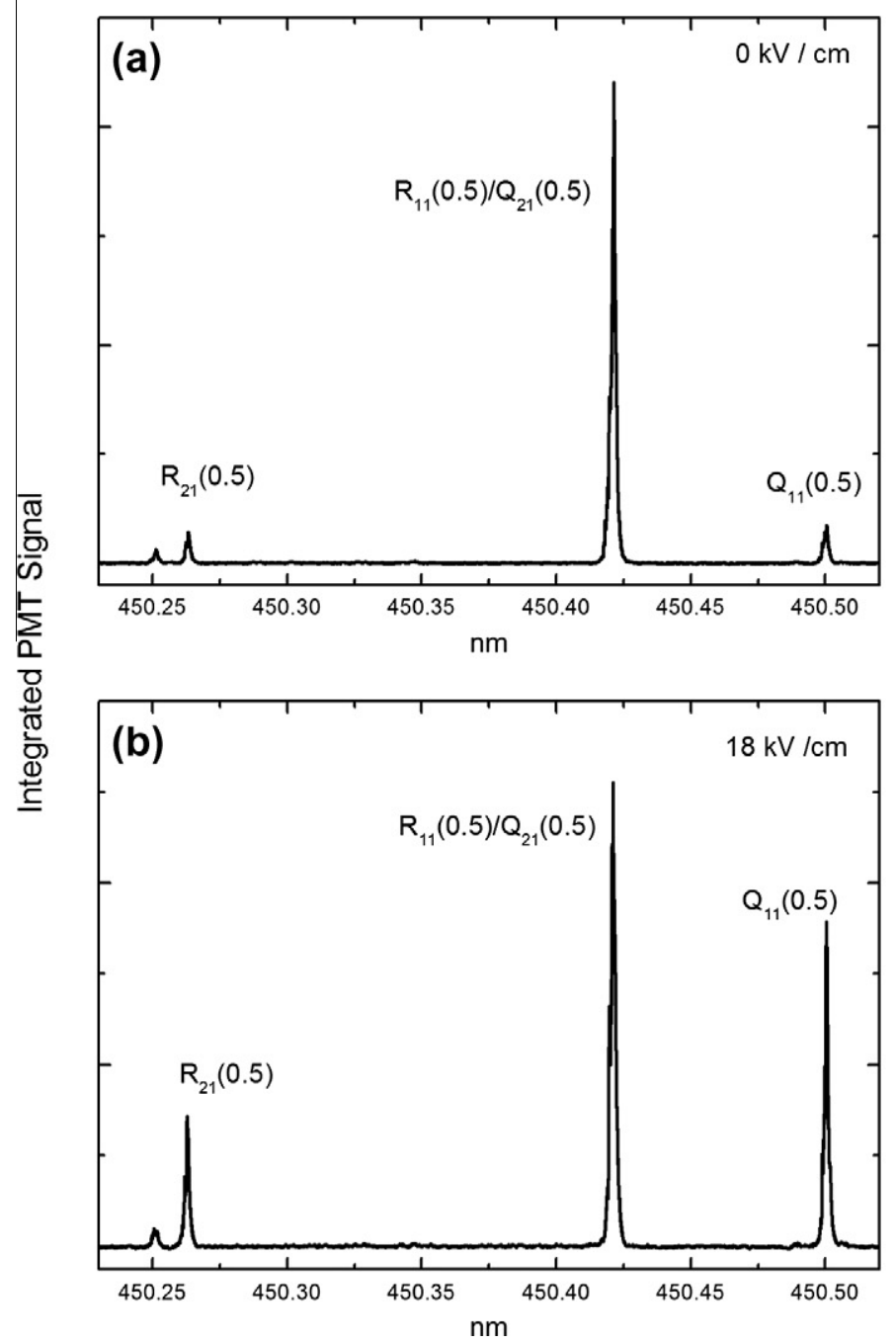

$f$-parity selected
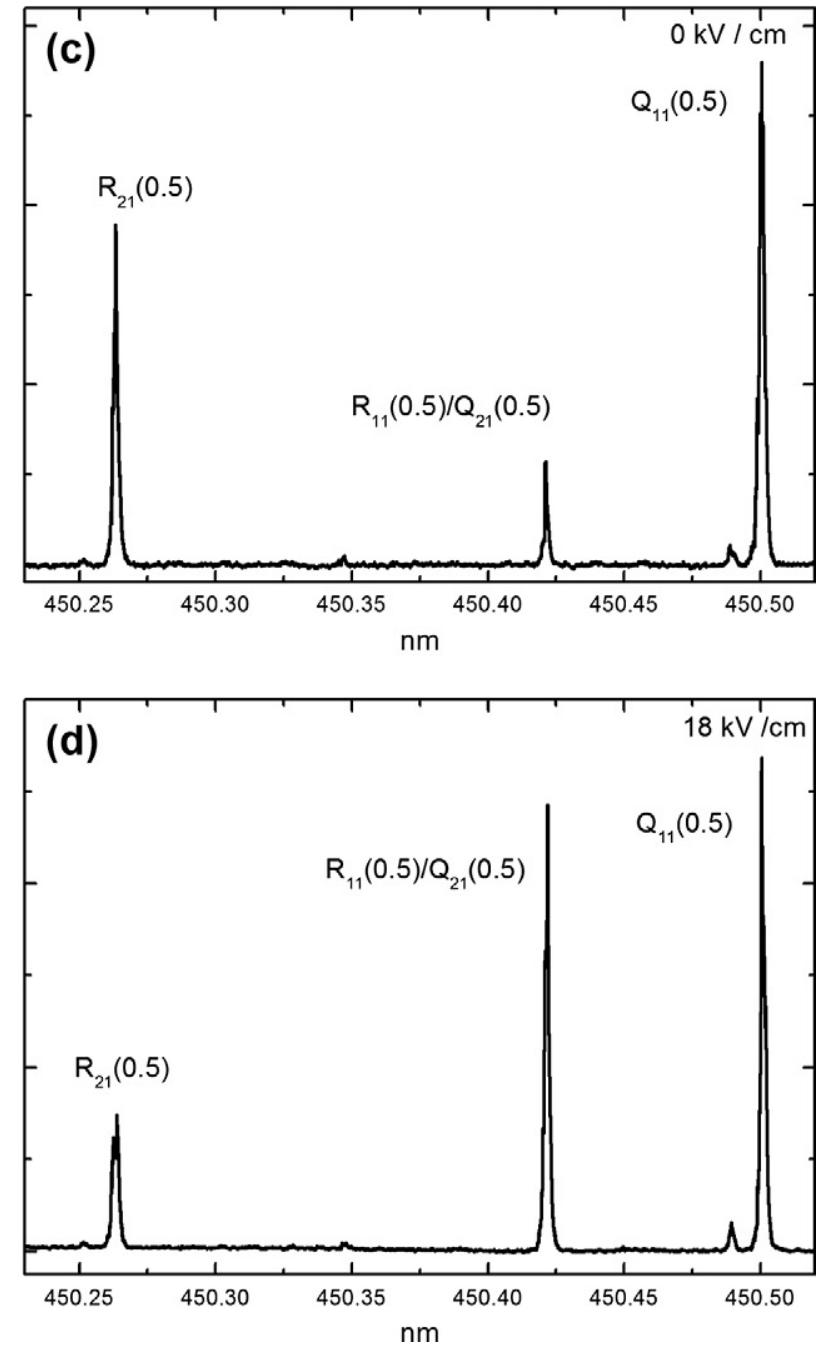

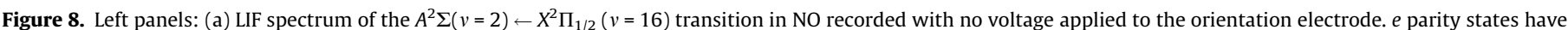

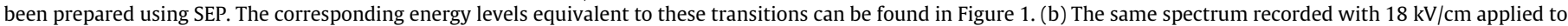

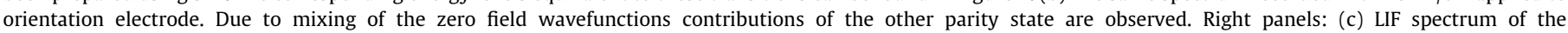

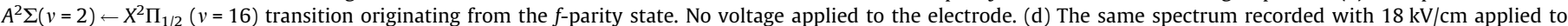
orientation electrode.

The dipole moment of NO $X^{2} \Pi_{1 / 2}\left(v^{\prime \prime}=16\right)$ has been measured [46]; however, no experimental determination for the zero field $\Lambda$-splitting is available for this vibrational state. Danielak et al. have reported zero field $\Lambda$-splitting for vibrational states ranging from $v=0$ to $v=7$ [45]. Within the precision of all known work of this kind, there is no demonstrable vibrational dependence to the $\Lambda$-splitting; therefore, we used a vibrationally averaged value for the $\Lambda$-splitting: $W_{\Lambda}=0.012 \mathrm{~cm}^{-1}$ for this analysis. In this way we may model the results of Figure 10 with no adjustable parameters. These simulations are plotted as solid lines in Figure 10. The excellent agreement with the experimentally derived results gives us confidence in the methods we have used in this Letter.

\section{Future frontiers: advantages and characteristics of the new approach}

The method of optical pumping followed by adiabatic orientation provides a new approach to orienting polar molecules in strong electric fields. In contrast to previous experiments of this kind, we employ optical state selection using a high resolution polarized laser instead of hexapole filtering in the first step of the experiment. Several interesting experimental opportunities are provided by this alternative approach.

\subsection{Orientation can be optically flipped: High field seekers can be used}

In contrast to multipole focusing, high field seeking states as well as low field seeking states can be orientated employing the present technique. Thus the orientation of the molecule can be optically switched, employing a static electric field, by tuning the laser back and forth between the transitions involved in parity state selection. This is particularly interesting for applications, where the electric field lines in the detection region are crucial for the experimental success, e.g. detecting charged particles (produced for example by REMPI) that are influenced by the electric field. Furthermore, shot-by-shot switching of the orientation is straightforward if the excitation wavelengths are close to each other as in this example, meaning that rapid signal averaging as a function of orientation can easily be automated. 

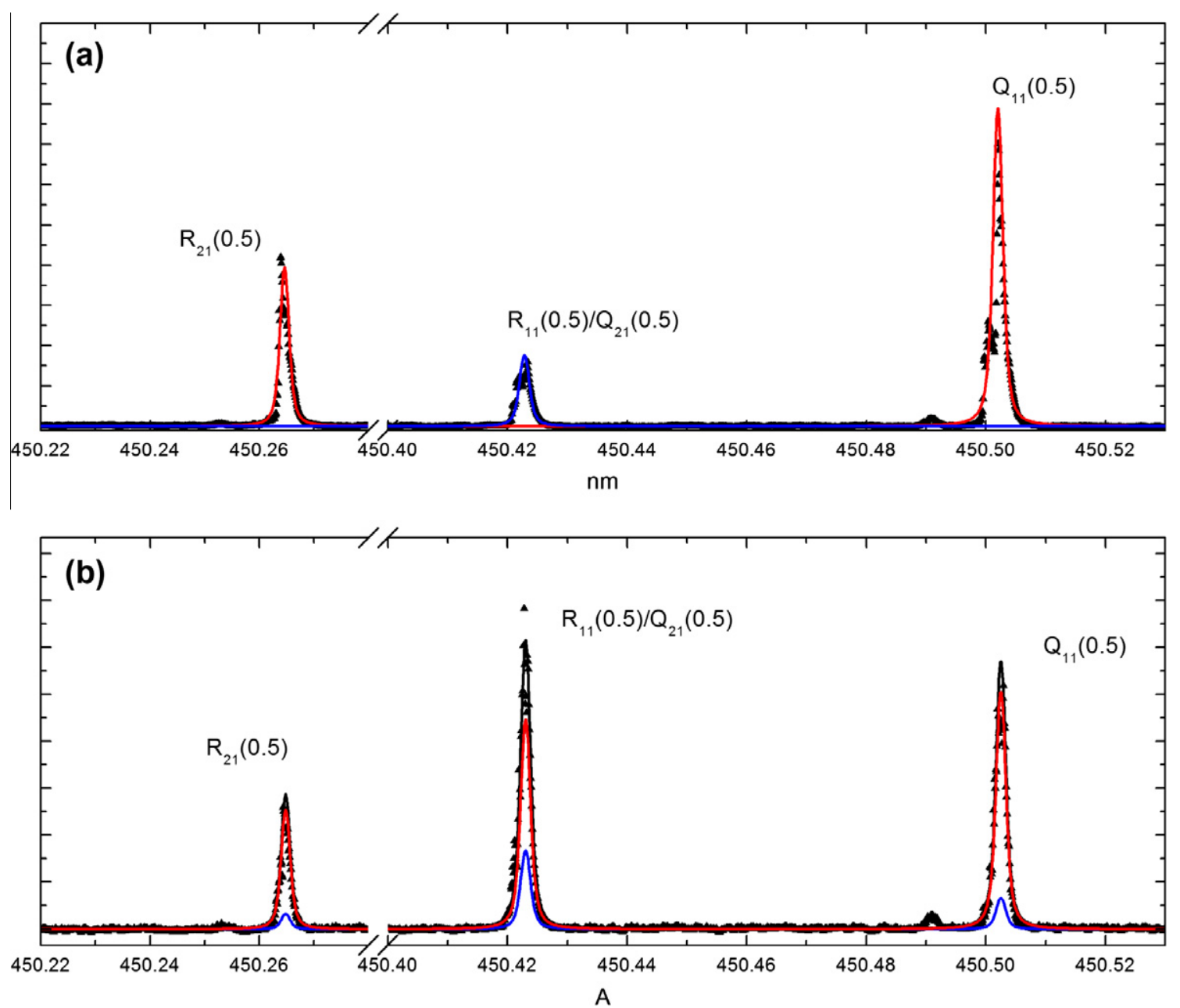

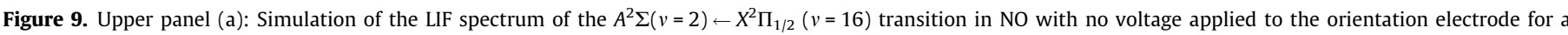

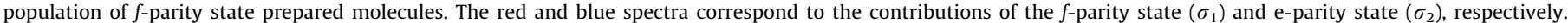

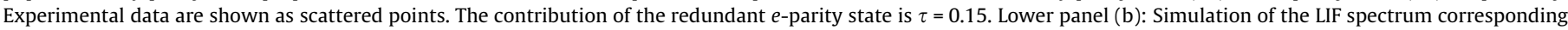

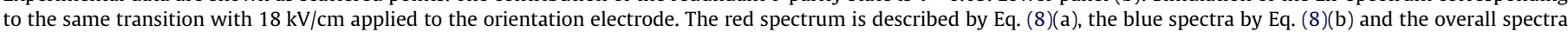
(black line) by Eq. (7). Experimental data are again shown as scattered points.

\subsection{The influence of sample velocity is limited}

In this Letter we have shown orientation of $\mathrm{NO}$ at a kinetic energy of $1000 \mathrm{meV}$. We next consider a Massey Criterion argument to understand the maximum kinetic energy that would be possible with this approach. Under our conditions we induced a Stark shift of about $0.07 \mathrm{~cm}^{-1}$ over a time scale of $70 \mu \mathrm{s}$. This corresponds to an energy ramp on the order of

$\left.\frac{d W_{\text {Stark }}}{d t}\right]_{\exp }=10^{3} \frac{\mathrm{cm}^{-1}}{\mathrm{~s}}$

as the molecule flies from the field free region into the region of orientation. The zero field $\Lambda$-splitting, $W_{\Lambda}=0.012 \mathrm{~cm}^{-1}=2.4 \times 10^{-25} \mathrm{~J}$ of this state can be used to define an uncertainty time scale.

$\delta \tau=\frac{h}{W_{\Lambda}}=\frac{6.63 \times 10^{-34} \mathrm{~J} \mathrm{~s}}{2.4 \times 10^{-25} \mathrm{~J}} \sim 2.8 \mathrm{~ns}$

From this one can calculate an energy ramp rate induced by an external electric field that is fast enough to induce optical (nonadiabatic) transitions between the $\Lambda$-doublets. This will be on the order of

$\left.\frac{d W_{\text {Stark }}}{d t}\right]_{\text {nonadiabatic }}=\frac{W_{\Lambda}}{\delta \tau}=\frac{2.4 \times 10^{-25} \mathrm{~J} \times 5 \times 10^{22} \frac{\mathrm{cm}^{-1}}{\mathrm{~J}}}{2.8 \mathrm{~ns}}=4 \times 10^{6} \frac{\mathrm{cm}^{-1}}{\mathrm{~s}}$.
This simple calculation serves only as an estimate; nevertheless, it shows that the velocity of the beam must be on the order of 1000 times higher than the highest used in this Letter before one would begin to detect nonadiabatic $\Lambda$-doublet transitions.

As long as we are within the adiabatic orientation limit, the velocity of the oriented molecules is no longer a defining aspect of the experiment. Hence, experiments can be carried out at high kinetic energies. Therefore, as discussed in the introduction to this Letter, potential steering effects of the colliding molecule in the approach to other molecules or to surfaces can be overcome. Furthermore, this suggests the astonishing possibility - to our knowledge the first - to prepare samples of oriented beams of polar ions. For example $\mathrm{HF}^{+}$possesses all of the necessary properties in its ground state that if one could optically prepare individual parity levels in a field free region, the states would adiabatically orient in a field of a few $\mathrm{kV} / \mathrm{cm}$. We return to this topic below.

\subsection{The experiment is smaller and simpler}

Another interesting characteristic of the new approach is the simplicity of the orientation electrode. One interested observer remarked that the complexity of the orientation has been transferred to the laser system. While this is certainly true, (a standard hexapole experiment needs no laser for preparing an oriented beam of molecules) lasers have become so common place in physical 


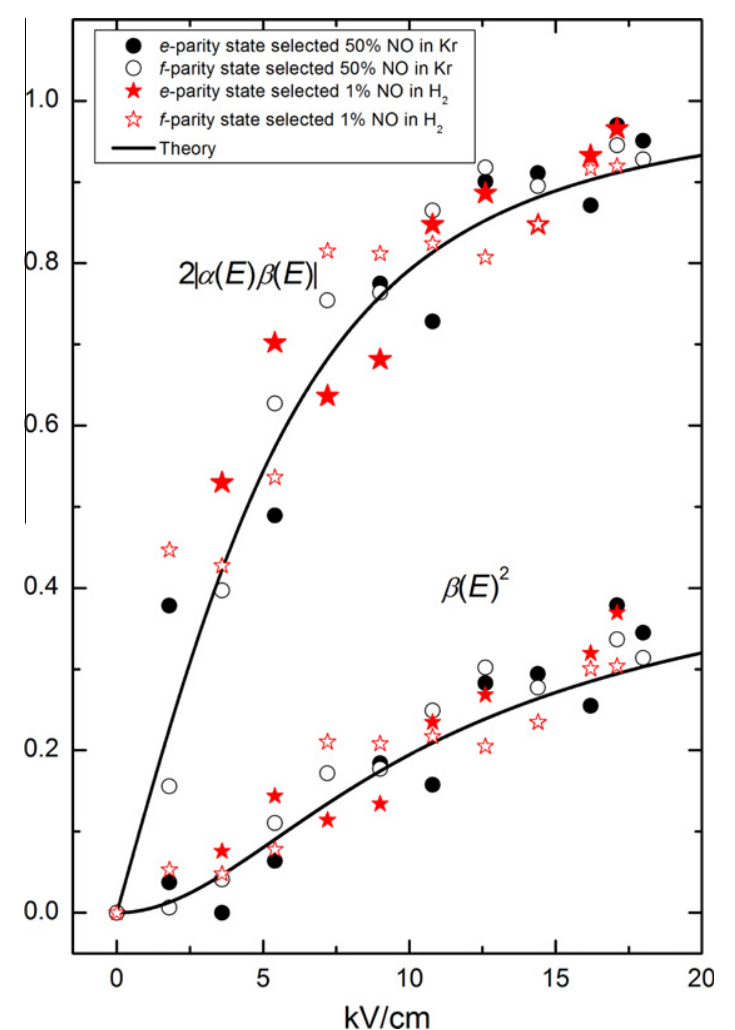

Figure 10. Square of the mixing coefficient $\beta(E)^{2}$ and the degree of orientation $2 \alpha(E) \beta(E)$ (as fraction of the maximum achievable degree of orientation) as a function of the electric field strength. Black solid circles denote $e$-parity state prepared molecules with $35 \mathrm{meV}$ translational energy, black open circles denote $f$ parity state prepared molecules with $35 \mathrm{meV}$ translational energy, red solid stars denote $e$-parity state prepared molecules with $1 \mathrm{eV}$ translational energy and red open stars denote $f$-parity state prepared molecules with $1 \mathrm{eV}$ translational energy. The solid lines show the calculated values following Eq. (5) and Eq. (9), respectively.

chemistry laboratories and optical pumping has become such a ubiquitous method that many might be willing to 'pay the price' or find that they already have!

For anyone already pumping molecular beams with a laser, the additional bother needed to implement this style of orientation is minimal. Figure 6 shows an image of the electrode used for orientation in this Letter, which was of course outfitted with a high voltage power supply and an appropriate HV feedthrough.

Moreover, the distance between the nozzle and the target can be kept quite short increasing the flux of oriented molecules on the sample. It is important to keep in mind that the method described in this Letter does not remove the portion of the beam that is not optically pumped. Unlike a hexapole, it does not physically filter the orientation states.

\subsection{Orientation achieved is limited only by Uncertainty principle}

In contrast to other methods like brute force orientation [47] and hexapole focusing of molecules with more complicated rotational structure (e.g. asymmetric tops), this approach produces a pure quantum state, so that more efficient orientation can be reached at lower electric field strengths. This is a significant advantage over the brute force method which has been theoretically compared to hexapole orientation [47]. Brute force techniques require much higher electric field strengths to achieve a similar degree of orientation. Here, a molecular beam passes through a pair of high electric field orientation electrodes without state selection. The high field mixes the rotational states, which leads to an anisotropic orientational distribution. Since this method relies on the second- and higher-order Stark effect, the dipole moment of the molecule should be large and the rotation constant should be small. Furthermore, state-to-state studies are excluded due to mixing of the different rotational states. A major advantage of brute force orientation over hexapole focusing, its relative generality, is reduced in importance in comparison to optical excitation with adiabatic orientation.

\subsection{The method can be extended to other systems}

The prospects for extending this approach to other molecules appear quite promising. In this section we take the liberty to speculate a bit about what we think should be possible. Figure 4 shows a schematic diagram of the basic idea behind this Letter. Here, an idealized two level system moves adiabatically from zero field (at the left) into a strong electric field (at the right). The molecule is assumed to have a zero field splitting, $\Delta_{1}$, and a 1 st order Stark effect resulting in an orientation field induced splitting, $\Delta_{2}$ In the case of the NO $X^{2} \Pi_{1 / 2}$ state, $\Delta_{1}$ refers to the $\Lambda$-splitting. Other quantum analogs include symmetric tops with inversion doubling $\left(\mathrm{NH}_{3}\right)$, linear molecules with bend mode vibrational excitation induced $l$-type doubling, and asymmetric tops with $K$-type doubling.

In general, it will be possible to find conditions where one or the other of these doublets can be optically pumped in a field free region, indicated by the solid red arrow. Under some conditions it may be advantageous to optically prepare the molecules in an electric pre-field (red dashed arrow), where the splitting is larger. Entering the electric field adiabatically, the states evolve into a high-field and a low-field seeker, corresponding to different orientations of the molecule with respect to the electric field lines.

We now outline the possibilities by describing conditions for optical pumping with adiabatic orientation for a number of examples.

\section{6. $\mathrm{CO}\left(a^{3} \Pi\right)$}

There are a number of molecules that are directly analogous to the NO molecule. $\mathrm{CO}\left(a^{3} \Pi\right.$ is one of them. Using a laser to directly excite the Cameron bands, ground state $\mathrm{CO}$ can be pumped to selectively produce states of defined parity $[38,48]$. The dipole moment of $\mathrm{CO}\left(a^{3} \Pi(1.3745 \mathrm{D})\right.$ is substantially larger than that of NO and the molecule exhibits a 1st order Stark effect [49]. Hence, lower electric field strengths are needed to achieve full orientation (ca. $4 \mathrm{kV} / \mathrm{cm}$ ). However, since both zero field $\Lambda$-doublet states are degenerate, each $\Lambda$-doublet correlates adiabatically to $|M|=1$ and 0 . See for example Figure 2 of Ref. [50]. Excitation with application of an external electric to lift this degeneracy or using circularly polarized light are two solutions to this problem, the first which has already been demonstrated in the laboratory [38].

$$
\text { 4.7. } \mathrm{OH}\left(\mathrm{X}^{2} \Pi_{\Omega}, v>0\right)
$$

$\mathrm{OH}$ in its electronic ground state also bears great quantum mechanical resemblance to NO. The $\Lambda$-splitting is resolvable [51] and the dipole moment is large [52]. While nearly any scheme for optical pumping is compatible with this orientation technique, infrared pumping (including overtone pumping) appears to be a particularly useful tool for extending this approach to other molecules. Here, narrow bandwidth laser pulses of high intensity in the IR are used to excite vibrational states with parity state selectivity. To make the point clear, we point out that individual parity states of NO $X^{2} \Pi_{1 / 2}(v=2$ and 3 ) have successfully been prepared using this technique in our laboratory [53]. Due to the large $\Lambda$-splitting of the $\mathrm{OH}$ radical, state selective excitation should even be easier than for the NO molecule. Pesce et al. recently measured the 
fundamental vibrational band of the $\mathrm{OH}$ radical at $3 \mu \mathrm{m}$ and easily resolved the $\Lambda$-splitting, which was on the order of a few $\mathrm{GHz}$ [54]. Hence we are confident, that this approach will be an attractive one for $\mathrm{OH}$ and many other molecules.

\section{8. $\mathrm{NH}_{3}(v>0)$}

Consider for instance the symmetric top molecule $\mathrm{NH}_{3}$ with a dipole moment of $1.5 \mathrm{D}$ [55]. Here, the rovibrational states of the electronic ground state exhibits inversion splitting due to the low barrier of the umbrella vibration. This splitting is in the order of $1 \mathrm{~cm}^{-1}$ in the vibrational ground state and $30 \mathrm{~cm}^{-1}$ in the first excited umbrella mode and easily resolved, for example in IR overtone [56] excitation. Both states have opposite parity and therefore behave like the $e$ - and $f$-parity states of the NO molecule: In an electric field the interaction of the dipole moment with the field leads to a Stark shift of both states resulting in an orientation of the molecule with respect to the electric field lines $\left(2 \mathrm{~cm}^{-1}\right.$ at $50 \mathrm{kV} / \mathrm{cm}$ ) [57]. Again, the prepared population of definite parity evolves adiabatically into the oriented states entering the electric field as depicted in Figure 4.

\section{9. $\operatorname{OCS}(v>0)$}

All points discussed above for NO are quantum mechanically transferable to linear molecules with bending vibrations. For example OCS with one quantum of bending excitation exhibits $l$ type doublets, which can be resolved with high resolution lasers in IR overtone and combination bands [58]. The large dipole moment of OCS (0.7 D) [59] ensures orientation will be complete at reasonable electric fields.

\subsection{0. $\mathrm{H}_{2} \mathrm{CO}$}

One of the most important implications of this Letter concerns orientation of near symmetric tops (asymmetric rotors). For these molecules the state selection of hexapole filters often becomes impractical as rotational resolution might no longer be possible [35-37]. The use of optical state selection again through infrared fundamental overtone or combination band excitation can overcome these difficulties. For at least one molecule, $\mathrm{CH}_{2} \mathrm{O}, \mathrm{SEP}$ is also possible [60].

Figure 11 show the rovibrational states of formaldehyde as a function of the electric field. This is a calculated Stark splitting diagram based on the work of Ref. [36] and may be compared to our idealized view shown in Figure 4. The Stark effect matrix elements are calculated in a prolate rotor basis set as demonstrated in Ref. [61]. The zero field states are split by an easily resolved $\sim 0.2 \mathrm{~cm}^{-1}$. Different $M$ states can be distinguished using circular polarized light for excitation. Alternatively one could excite the molecule in the presence of an external electric field and spectrally resolve the individual $M$-states similar to the method described in Ref. [38]. As one can see, some states evolve adiabatically into pure low field seekers and others into high field seekers, which means the molecule becomes oriented.

\subsection{1. $H F^{+}$}

Another interesting feature of this method is its possible application to orient charged molecules in electronic $\Pi$-states. Due to its charge, focusing an ion with multipole lenses is impossible, and therefore no pure quantum state can be selected by means of a hexapole filter. This obstacle can easily be overcome using optical state selection methods; however, the orientation field in front of the surface is still needed. All effects induced by this field like acceleration and deflection should be carefully considered by the

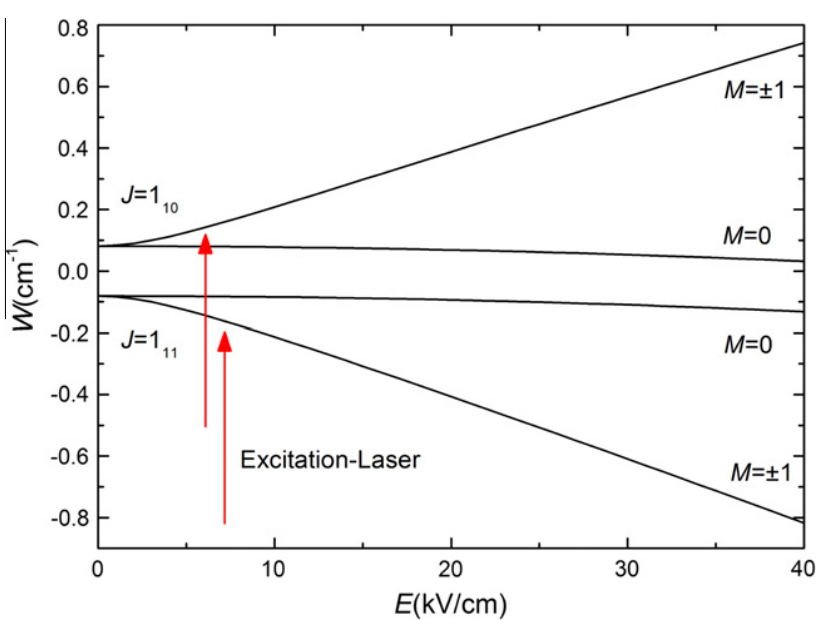

Figure 11. Stark energy curves for the $J=1_{10}$ and $J=1_{11}$ states of the asymmetric top molecule $\mathrm{H}_{2} \mathrm{CO}$. Employing an elegant excitation scheme, populations of high field seeking states/low field seeking states can be prepared. The red arrows assign the position of the excitation laser to prepare different orientation states.

experimentalist, nevertheless we are confident that the technique presented here offers a unique opportunity to orient even charged molecules exhibiting a strong $\Lambda$-splitting like $\mathrm{HF}^{+}$in its electronic ground state $X^{2} \Pi_{1 / 2}[62]$.

\section{Conclusion}

Highly vibrationally excited $\mathrm{NO}(v=16)$ molecules have been oriented using the Stark effect of diatomic molecules. For the first time, the arrangement of optical pumping in a region free of electric fields followed by adiabatic transport into a static electric field has been applied to this kind of experiments. It is shown that almost full orientation is achieved at electric field strengths of $18 \mathrm{kV} / \mathrm{cm}$ and no kinetic energy dependence on the degree of orientation is observed. We demonstrate that this approach is a promising alternative to the commonly used hexapole filter technique for state selection, extendable to many other molecules.

\section{Acknowledgement}

AMW and XMY would like to acknowledge the Alexander von Humboldt Foundation for support for this Letter.

\section{Appendix A. Supplementary data}

Supplementary data associated with this article can be found, in the online version, at http://dx.doi.org/10.1016/ j.cplett.2012.03.056.

\section{References}

[1] M.G. Tenner, E.W. Kuipers, A.W. Kleyn, S. Stolte, J. Chem. Phys. 94 (1991) 5197.

[2] F.H. Geuzebroek, A.E. Wiskerke, M.G. Tenner, A.W. Kleyn, S. Stolte, A. Namiki, J. Phys. Chem. 95 (1991) 8409.

[3] M. Okada, S. Goto, T. Kasai, J. Phys. Chem. C 112 (2008) 19612.

[4] M. Hashinokuchi, M. Okada, H. Ito, T. Kasai, K. Moritani, Y. Teraoka, Phys. Rev. Lett. 100 (2008).

[5] G.H. Fecher, N. Bowering, M. Volkmer, B. Pawlitzky, U. Heinzmann, Surf. Sci. 230 (1990) L169.

[6] M. Brandt, T. Greber, N. Bowering, U. Heinzmann, Phys. Rev. Lett. 81 (1998) 2376.

[7] M. Okada, S. Goto, T. Kasai, Phys. Rev. Lett. 95 (2005).

[8] U. Heinzmann, S. Holloway, A.W. Kleyn, R.E. Palmer, K.J. Snowdon, J. Phys.: Condens. Matter 8 (1996) 3245.

[9] C. Bartels, R. Cooper, D.J. Auerbach, A.M. Wodtke, Chem. Sci. 2 (2011) 1647.

[10] A.M. Wodtke, D. Matsiev, D.J. Auerbach, Prog. Surf. Sci. 83 (2008) 167. 
[11] A.M. Wodtke, J.C. Tully, D.J. Auerbach, Int. Rev. Phys. Chem. 23 (2004) 513.

[12] I. Rahinov, R. Cooper, D. Matsiev, C. Bartels, D.J. Auerbach, A.M. Wodtke, Phys. Chem. Chem. Phys. 13 (2011) 12680.

[13] K. Lacmann, D.R. Herschbach, Chem. Phys. Lett. 6 (1970) 106.

[14] J.W. Gadzuk, J.K. Norskov, J. Chem. Phys. 81 (1984) 2828.

[15] A.W. Kleyn, J. Los, E.A. Gislason, Phys. Rep. Rev. Sect. Phys. Lett. 90 (1982) 1

[16] S. Roy, N.A. Shenvi, J.C. Tully, J. Chem. Phys. 130 (2009) 174716

[17] N. Shenvi, S. Roy, J.C. Tully, J. Chem. Phys. 130 (2009) 174107.

[18] Y.H. Huang, C.T. Rettner, D.J. Auerbach, A.M. Wodtke, Science 290 (2000) 111

[19] N. Shenvi, S. Roy, J.C. Tully, Science 326 (2009) 829.

[20] X. Yang, J.M. Price, J.A. Mack, C.G. Morgan, C.A. Rogaski, D. McGuire, E.H. Kim, A.M. Wodtke, J. Phys. Chem. 97 (1993) 3944.

[21] M. Silva, R. Jongma, R.W. Field, A.M. Wodtke, Annu. Rev. Phys. Chem. 52 (2001) 811.

[22] A. Gross, S. Wilke, M. Scheffler, Phys. Rev. Lett. 75 (1995) 2718.

[23] J.T. Kindt, J.C. Tully, Surf. Sci. 477 (2001) 149.

[24] D.O.N. Gardner, A. Al-Halabi, G.J. Kroes, Chem. Phys. Lett. 376 (2003) 581.

[25] H.F. Busnengo, E. Pijper, G.J. Kroes, A. Salin, J. Chem. Phys. 119 (2003) 12553.

[26] H.G. Bennewitz, W. Paul, C. Schlier, Z. Angew. Phys. 141 (1955) 6.

[27] K.H. Kramer, R.B. Bernstein, J. Chem. Phys. 42 (1965) 767.

[28] J.E. Mosch, S.A. Safron, J.P. Toennies, Chem. Phys. 8 (1975) 304

[29] H.G. Bennewitz, K.H. Kramer, J.P. Toennies, W. Paul, Z. Angew. Phys. 177 (1964) 84.

[30] J.T. Hougen, Methane Symmetry Operations, 2009, <http://www.nist.gov/pml/ pubs/methane/index.cfm>

[31] This neglects the possible influence of hyperfine depolarization.

[32] E.W. Kuipers, M.G. Tenner, A.W. Kleyn, S. Stolte, Chem. Phys. 138 (1989) 451

[33] M.G. Tenner, E.W. Kuipers, W.Y. Langhout, A.W. Kleyn, G. Nicolasen, S. Stolte Surf. Sci. 236 (1990) 151

[34] M. Okada, T. Kasai, Eur. Phys. J. B 75 (2010) 71.

[35] E.M. Jones, P.R. Brooks, J. Chem. Phys. 53 (1970) 55.

[36] T.D. Hain, R.M. Moision, T.J. Curtiss, J. Chem. Phys. 111 (1999) 6797.

[37] D.C. Che, F. Palazzetti, Y. Okuno, V. Aquilanti, T. Kasai, J. Phys. Chem. A 114 (2010) 3280.

[38] M. Drabbels, S. Stolte, G. Meijer, Chem. Phys. Lett. 200 (1992).

[39] D. Matsiev, J. Chen, M. Murphy, A.M. Wodtke, J. Chem. Phys. 118 (2003) 9477

[40] J. Chen, D. Matsiev, J.D. White, M. Murphy, A.M. Wodtke, Chem. Phys. 301 (2004) 161.

[41] L. Velarde, D.P. Engelhart, D. Matsiev, J. LaRue, D.J. Auerbach, A.M. Wodtke, Rev. Sci. Instrum. 81 (2010)

[42] J.D. White, J. Chen, D. Matsiev, D.J. Auerbach, A.M. Wodtke, J. Chem. Phys. 124 (2006).

[43] M. Drabbels, A.M. Wodtke, Chem. Phys. Lett. 256 (1996) 8

[44] M.J.L. de Lange, J.J. van Leuken, M. Drabbels, J. Bulthuis, J.G. Snijders, S. Stolte, Chem. Phys. Lett. 294 (1998) 332.

[45] J. Danielak, U. Domin, R. Kepa, M. Rytel, M. Zachwieja, J. Mol. Spectrosc. 181 (1996) 394

[46] M. Drabbels, A.M. Wodtke, J. Chem. Phys. 106 (1997) 3024.

[47] J. Bulthuis, J.J. Vanleuken, S. Stolte, J. Chem. Soc., Faraday Trans. 91 (1995) 205

[48] R.W. Field, S.G. Tilford, R.A. Howard, J. Mol. Spectrosc. 44 (1972) 347.

[49] B.G. Wicke, R.W. Field, W. Klemperer, J. Chem. Phys. 56 (1972).

[50] R.T. Jongma, T. Rasing, G. Meijer, J. Chem. Phys. 102 (1995).

[51] J.P. Maillard, J. Chauville, A.W. Mantz, J. Mol. Spectrosc. 63 (1976) 120.

[52] W.L. Meerts, A. Dynamus, Chem. Phys. Lett. 23 (1973).

[53] (to be published).

[54] G. Pesce, G. Rusciano, A. Sasso, Chem. Phys. Lett. 374 (2003) 425

[55] C.H. Townes, A.L. Schwlow, Microwave Spectroscopy, Dover, New York, 1975.

[56] S. Höjer, H. Ahlberg, A. Rosén, Appl. Phys. B 52 (1991) 200.

[57] Y.T. van de Meerakker, H.L. Bethlem, N. Vanhaecke, G. Meijer, Chem. Rev., submitted (2011).

[58] K. Tanaka, H. Ito, T. Tanaka, J. Mol. Spectrosc. 107 (1984) 324

[59] K. Tanaka, H. Ito, K. Harada, T. Tanaka, J. Chem. Phys. 80 (1984) 5893.

[60] D.E. Reisner, P.H. Vaccaro, C. Kittrell, R.W. Field, J.L. Kinsey, H.L. Dai, J. Chem. Phys. 77 (1982)

[61] D.J. Coffey, C. Yamada, E. Hirota, J. Mol. Spectrosc. 64 (1977) 98

[62] S. Gewurtz, H. Lew, P. Flainek, Can. J. Phys. 53 (1975).

[63] J. Reuss, State Selection by nonoptical methods, in: G. Scoles (Ed.), Atomic and Molecular Beam methods, Oxford University Press, New York, Oxford, 1988.

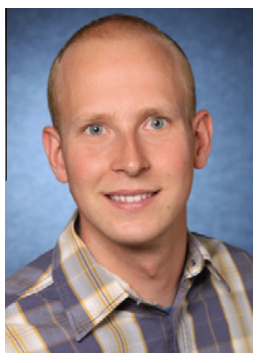

Nils Bartels received his BSc (2009) and MSc (2011) degree in Chemistry from the University of Göttingen. He joined the group of Alec Wodtke for his master thesis and started his PhD last year. He built the injectionseeded OPO used in this Letter from scratch and is now studying steric effects in gas-surface scattering. Another project of interest to him focuses on the production of highly vibrationally excited CO using a novel variant of stimulated emission pumping.

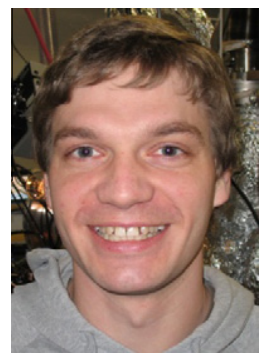

Nils Hocke has been working as an undergraduate student in Alec Wodtke's group since 2011. He is presently pursuing his Diplom.

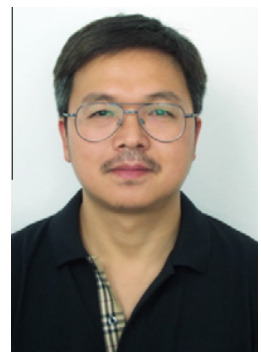

Xueming Yang received his PhD (1986) at the University of California, Santa Barbara. After postdocs at Princeton and Berkeley, he was appointed research fellow (1995) at the Institute for Atomic and Molecular Sciences (Taipei), later becoming a full research fellow. He moved to the Dalian Institute of Chemical Physics (2004) becoming Director of the State key laboratory of Molecular Reaction Dynamics. He was elected to the Chinese Academy of Science in 2011. He is best known for his work in the field of gas-phase chemical dynamics, especially exploiting Rydberg-atom tagging to study simple bimolecular and photochemical reactions.

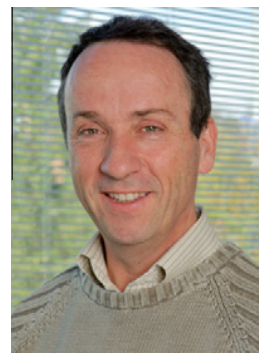

Alec M. Wodtke received his BA degree (1981) from the University of Utah in Chemistry. He obtained his PhD degree (1986) in Physical Chemistry from UC Berkeley. From 1988-2010, he was on the faculty of the Department of Chemistry \& Biochemistry at the University of California, Santa Barbara. In 2010, he was awarded the Alexander von Humboldt Professorship, which he assumed at the Georg-August University (Göttingen), also simultaneously being appointed as a director at the Max Planck Institute for Biophysical Chemistry. He is known for his work in the field of experimental chemical dynamics, especially concerning vibrationally excited molecules on surfaces.

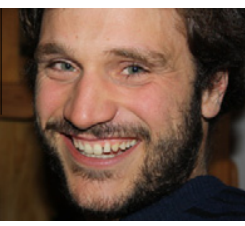

Tim Schäfer received his $\mathrm{PhD}$ in 2009 from the University of Göttingen in Chemistry, studying the vibrational energy relaxation of hydrogen bonded liquids with femtosecond mid-infrared spectroscopy under the supervision of Dirk Schwarzer in the group of Jürgen Troe. After a post-doc position with Alec Wodtke in Santa Barbara (2009/2010) he is now back in Göttingen, where his current research focuses on steric effects of energy relaxation of highly vibrational excited molecules in collisions with metal surfaces. 